\title{
Optical Characterization of Graphene and Highly Oriented Pyrolytic Graphite
}

\author{
R. Kostić, M. Mirić*, T. Radić, M. Radović, R. Gajić and Z.V. Popović \\ Institute of Physics, Center for Solid State Physics and New Materials, Belgrade, Serbia
}

\begin{abstract}
We present a symmetry analysis of allowed infrared and Raman modes in graphene and highly oriented pyrolytic graphite. Surface structure for highly oriented pyrolytic graphite is examined using atomic force microscopy. As experimental tools, we used infrared spectroscopic ellipsometry in order to investigate the pseudodielectric function of highly oriented pyrolytic graphite in the mid-infrared range $\left(500-7000 \mathrm{~cm}^{-1}\right)$ and Raman spectroscopy to investigate the influence of layers number decrease. As a result, we propose a method for an experimental verification of graphene.
\end{abstract}

PACS numbers: 63.20.D-, 78.67.Wj, 81.05.U-

\section{Introduction}

Highly oriented pyrolytic graphite (HOPG) is artificially grown graphite with an almost perfect alignment perpendicular to the carbon planes in an $\mathrm{ABAB}$ manner. Graphite is built from hexagonal planes of carbon atoms. Almost all chemical bonding in graphite is associated with the strong $s p^{2}$ intralayer bonds and layers are coupled by very weak van der Waals bonds. As a result of this strongly anisotropic bonding there is weak electron and phonon dispersion along the direction perpendicular to the carbon planes. Because the interaction between layers is very weak, behavior of a single hexagonal plane of carbon atoms (graphene) is crucial for all physical properties of graphite.

Experimental success in realizing well-ordered ultrathin graphite films, consisting of a few $(n)$ graphene layers ( $n \mathrm{GLs})$, gives possibility to investigate the properties of graphene [1-4].

In this paper we review the vibrational properties of graphene and graphite to compare them to our results of infrared spectroscopic ellipsometry for HOPG and Raman spectroscopy for HOPG and a few-layers graphene samples. We review the symmetry of graphene and graphite, symmetry of phonon branches and selection rules.

HOPG sample is provided by Omicron. Surface topography measurements of HOPG were performed on Omicron UHV VT AFM/STM system operating in contact AFM mode under ultrahigh vacuum on room temperature, and presented in Fig. 1.

Sample was further treated by cleaving sample layers along $a b$-plane with adhesive tape. Graphene is obtained by mechanical exfoliation of $\mathrm{HOPG}$ layers onto $\mathrm{SiO}_{2} / \mathrm{Si}$ substrate.

\footnotetext{
* corresponding author; e-mail: mmiric@ipb.ac.rs
}

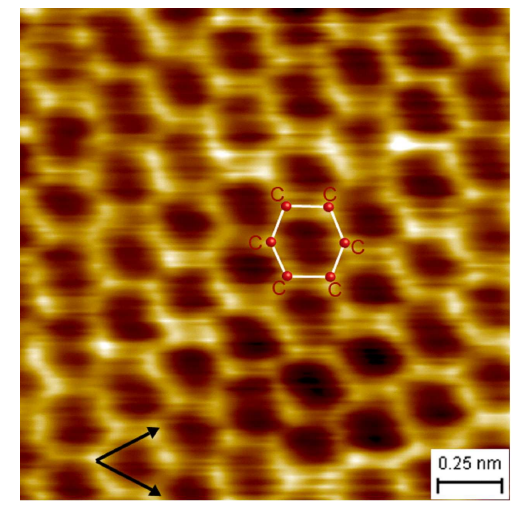

Fig. 1. HOPG, atomic resolution, AFM contact mode.

\section{Symmetry and selection rules}

The point group symmetry of ideal single graphene layer is $D_{6 h}$. There are two equivalent atoms per unit cell, giving six normal modes for the zone center. The irreducible representations for the zone center modes of a single layer are given by: $\Gamma^{\text {graphene }}=A_{2 \mathrm{u}}+B_{2 \mathrm{~g}}+E_{1 \mathrm{u}}+E_{2 \mathrm{~g}}$. Single layer vibrational modes properties (assignation, activity, energy) are summarized in Table. The $A_{2 u}$ represents translation perpendicular to the graphene plane and $E_{1 \mathrm{u}}$ represents the translation in the graphene plane. The $B_{2 \mathrm{~g}}$ mode is an inactive optical phonon where the carbon atoms move perpendicularly to the graphene plane. The $E_{2 \mathrm{~g}}$ mode is doubly degenerate Raman active optical vibration, where the carbon atoms move in graphene plane. In the first-order optical spectrum of graphene we expect only one Raman active $E_{2 \mathrm{~g}}$ optical mode at $\approx 1587 \mathrm{~cm}^{-1}[1,4]$.

Inside the Brillouin zone along the in-plane high-symmetry directions $\Gamma-M(\Sigma)$ and $\Gamma-K-M(T)$ the point group symmetry is reduced to $C_{2 v}$. In-plane optical and acoustic phonons split into two non-degenerated 
modes which belong to $A_{1}$ and $B_{1}$ in the molecular notation i.e. representation of $C_{2 v}$ group. More precisely, along $\Gamma-M(\Sigma)$ direction the LO and LA phonons transform according to $\Sigma_{1}\left(A_{1}\right)$ and TO and TA phonons according to $\Sigma_{3}\left(B_{1}\right)$. Along $\Gamma-K-M(T)$ direction the TO and LA branches belong to $T_{1}\left(A_{1}\right)$ and the LO and TA branches to $T_{3}\left(B_{1}\right)$. Point group $D_{6 h}$ reduces to $D_{3 h}$ at $K$ and to $D_{2 h}$ at the $M$ point of the Brillouin zone. At all high-symmetry points $\Gamma, K$ and $M$ the phonon eigenvectors of graphene are completely given by symmetry [5-8].

Graphite, the full three-dimensional lattice built from graphene layers, contains four atoms in the unit cell. Graphite belongs to the $D_{6 h}^{4}\left(P 6_{3} / m m c\right)$ space group. Its isogonal point group is $D_{6 h}$. The irreducible representations for the zone center modes of graphite are given by: $\Gamma^{\text {graphite }}=2 A_{2 \mathrm{u}}+2 B_{2 \mathrm{~g}}+2 E_{1 \mathrm{u}}+2 E_{2 \mathrm{~g}}$. Graphite vibrationalmodes properties are summarized in Table. Graphite crystal has two infrared (IR) $\left(E_{1 \mathrm{u}}\right.$ at $\approx 1587 \mathrm{~cm}^{-1}$ and $A_{2 \mathrm{u}}$ at $\left.\approx 870 \mathrm{~cm}^{-1}\right)$ and two Ra$\operatorname{man}\left(E_{2 \mathrm{~g}(2)}\right.$ at $\approx 1582 \mathrm{~cm}^{-1}$ and $E_{2 \mathrm{~g}(1)}$ at $\left.\approx 42 \mathrm{~cm}^{-1}\right)$ active vibrations at the $\Gamma$ point of the Brillouin zone. In the early literature on the Raman scattering in graphite the $E_{2 \mathrm{~g}(2)}$ mode at $\approx 1582 \mathrm{~cm}^{-1}$ was usually called the $G$ peak, Table. Origin of the IR active $E_{1 \mathrm{u}}$ and Raman active $E_{2 \mathrm{~g}(2)}$ modes in graphite is Raman active $E_{2 \mathrm{~g}}$ mode in graphene.

If number of layers increases from graphene $(n=1)$ to graphite $(n=\infty)$, frequency of $G$-band decreases from $1587 \mathrm{~cm}^{-1}$ to $1581.5 \mathrm{~cm}^{-1}$ while $G$-band intensity increases over the range $n=1$ to $n=20$ almost linearly [1]. Frequency and intensity of $G$-mode (Raman active $E_{2 \mathrm{~g}}$ mode) is determined by the number of graphene layers. Origin of the Raman active $E_{2 \mathrm{~g}(1)}$ mode is acoustic $E_{1 \mathrm{u}}$ mode of graphene. We must emphasize that low-frequency Raman active mode $\left(E_{2 \mathrm{~g}(1)}\right)$ at $\approx 42 \mathrm{~cm}^{-1}$ is very low intensity mode and difficult to be registered by the Raman scattering. It is usually identified by neutron scattering. According to selection rules this mode is present in graphite and absent in graphene Raman spectra, but it practically cannot be used as method for an experimental verification of graphene.

TABLE

Symmetry and selection rules for first-order spectrum.

\begin{tabular}{|c|c|c|c|c|c|c|c|c|}
\hline $\begin{array}{c}\text { Graphene } \\
2 \mathrm{D} \text { (two } \\
\text { atoms per } \\
\text { primitive cell) } \\
D_{6 h} \\
\end{array}$ & \multicolumn{2}{|c|}{$\begin{array}{c}E_{2 \mathrm{~g}} \\
\text { Raman } \\
\approx 1587 \mathrm{~cm}^{-1} \\
\text { in plane }\end{array}$} & \multicolumn{2}{|c|}{$\begin{array}{c}B_{2 \mathrm{~g}} \\
\text { silent } \\
\approx 870 \mathrm{~cm}^{-1} \\
\text { out of plane }\end{array}$} & \multicolumn{2}{|c|}{$\begin{array}{c}E_{1 \mathrm{u}} \\
\text { acoustic } \\
\left(T_{x}, T_{y}\right)\end{array}$} & \multicolumn{2}{|c|}{$\begin{array}{c}A_{2 \mathrm{u}} \\
\text { acoustic } \\
\left(T_{z}\right)\end{array}$} \\
\hline $\begin{array}{c}\text { Graphite } \\
3 \mathrm{D} \text { (four } \\
\text { atoms per } \\
\text { primitive cell) } \\
D_{6 h}^{4}\end{array}$ & $\begin{aligned} & E_{1 \mathrm{u}} \\
& \mathrm{IR} \\
& \approx 1587 \\
& \mathrm{~cm}^{-1}\end{aligned}$ & $\begin{array}{c}E_{2 \mathrm{~g}(2)} \\
\operatorname{Raman} \\
\approx 1582 \\
\mathrm{~cm}^{-1} \\
G \text {-band }\end{array}$ & $\begin{array}{l}B_{2 \mathrm{~g}(2)} \\
\text { silent } \\
\approx 870 \\
\mathrm{~cm}^{-1}\end{array}$ & $\begin{aligned} & A_{2 \mathrm{u}} \\
& \mathrm{IR} \\
& \approx 868 \\
& \mathrm{~cm}^{-1}\end{aligned}$ & $\begin{array}{c}E_{2 \mathrm{~g}(1)} \\
\operatorname{Raman} \\
\approx 42 \\
\mathrm{~cm}^{-1}\end{array}$ & $\begin{array}{c}E_{1 \mathrm{u}} \\
\text { acoustic } \\
\left(T_{x}, T_{y}\right)\end{array}$ & $\begin{array}{l}B_{2 \mathrm{~g}(1)} \\
\text { silent } \\
\approx 127 \\
\mathrm{~cm}^{-1}\end{array}$ & $\begin{array}{c}A_{2 \mathrm{u}} \\
\text { acoustic } \\
\left(T_{z}\right)\end{array}$ \\
\hline
\end{tabular}

If scattered light is collected from disordered part of the graphite sample, a new Raman line appears at $\approx 1370 \mathrm{~cm}^{-1}$ [1]. This mode was first reported by Tunistra [9], and named $D$-mode for disorder induced mode. Since zone-boundary phonons do no satisfy the Raman fundamental selection rule, they are not seen in first order Raman spectra of defect-free graphite. In this paper we will follow the convention where $D$ stands for modes coming approximately from the $K$ point of graphite Brillouin zone.

In second-order spectrum of graphite there are two main features. The first one is moderate to strong mode at $\approx 2700 \mathrm{~cm}^{-1}$, historically denoted as: $D^{*}$ or $2 D$ [4] mode (frequency is close to twice $D$ mode frequency) or $G^{\prime}$ mode (since it is the second most prominent peak in graphite). The second one is moderate mode at $\approx 3248 \mathrm{~cm}^{-1}$ (denoted as $G^{*}$ mode). Many other much weaker Raman modes were also reported, like weak intensity combination scattering at $\approx 2450 \mathrm{~cm}^{-1}$.

$D^{*}$ is clearly not an overtone of the Raman-allowed first-order phonon spectra. $D^{*}$ feature frequency is very close to twice the $D$ mode frequency. The frequency of the $D$ mode shifts to higher energies with increasing excitation energies. The shift was observed to be linear in wide range of excitation energies, and the $D^{*}$ band has twice the $D$ band slope, confirming the $D^{*}$ assignment as an overtone. The excitation energy dependence of the $D^{*}$ band frequencies and other effects are in agreement to concept of a double resonant process that selectively enhances a particular phonon wave vector and hence phonon energy [6]. Selected phonon wave vector is close to the $K$ point of the graphite Brillouin zone. 
This approach predicts that graphene has a single $D^{*}$ peak. As number of layers increases structure of the $D^{*}$ becomes more and more complicate and the center of the feature moves towards higher frequencies [1-3]. Position and structure of $D^{*}$ band reflects number of graphene layers in the sample.

$G^{*}$-peak is associated with the overbending of the longitudinal optical branches of graphite, as the LO branch has its maximum not far from the $\Gamma$ point but inside the Brillouin zone, in contrast to most other materials.

\section{Optical characterization}

Ellipsometry measures the change in polarization state of light as it reflects from a sample surface. That change is expressed as the ratio of the complex Fresnel reflection coefficients $r_{p}$ and $r_{s}$ (for light polarized parallel and perpendicular to the plane of incidence, respectively). That ratio is: $\rho=r_{p} / r_{s}=\tan (\Psi) \mathrm{e}^{\mathrm{i} \Delta}$, where $\tan (\Psi)$ is the ratio of the magnitudes of $r_{p}$ and $r_{s}$ and $\Delta$ is the phase difference between the coefficients. Values for $\Psi$ and $\Delta$ at each wavelength comprise the ellipsometric spectra.

Our ellipsometric measurements of HOPG were performed using SOPRA Fourier Transform Infra-Red Spectroscopic Ellipsometer in the $650-7000 \mathrm{~cm}^{-1}$ range, with spectral resolution of $2 \mathrm{~cm}^{-1}$. In this paper we present measurements with an incidence angle $\theta=80^{\circ}$.

In Fig. 2, both real and imaginary parts of the pseudodielectric function are presented. In experimental spectra we identified both IR active modes predicted by symmetry analysis. For the oblique incidence, both IR active modes, $E_{1 \mathrm{u}}$ "in-plane" mode on $\approx 1587 \mathrm{~cm}^{-1}$, and $A_{2 \mathrm{u}}$ "out-of-plane" mode on $\approx 868 \mathrm{~cm}^{-1}$, contribute to the pseudodielectric function. Reflectivity measurements of HOPG, Nemanich [10], in two different polarizations are given in the inset. The ordinary component of dielectric function has classic quasi-free-carrier behavior, and the imaginary part $\left\langle\varepsilon_{\mathrm{i}}\right\rangle$ increases with decreasing energy, while the real part $\left\langle\varepsilon_{\mathrm{r}}\right\rangle$ decreases with decreasing energy.

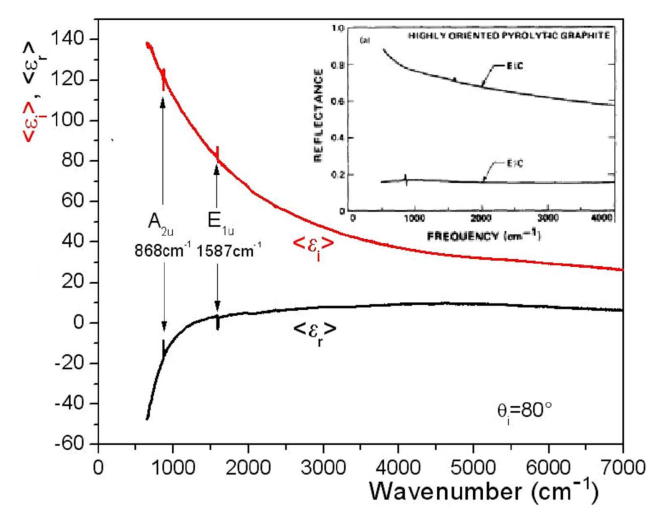

Fig. 2. Real $\left\langle\varepsilon_{\mathrm{r}}\right\rangle$ and imaginary $\left\langle\varepsilon_{\mathrm{i}}\right\rangle$ parts of pseudodielectric function of HOPG in mid-infrared range. Inset: IR reflectivity spectra from [10].
IR activity is a consequence of the three-dimensional structure of graphite, Table. In single layer, with two equivalent atoms per unit cell, there are no IR active modes.

The Raman scattering measurements were performed at room temperature using the Jobin-Yvon T64000 triple spectrometer system equipped with a liquid-nitrogen cooled CCD detector. The $\lambda=514.5 \mathrm{~nm}$ line of an $\mathrm{Ar}^{+}$ laser was used as an excitation source.

In Fig. 3 we present the Raman spectra of our HOPG samples. The most interesting frequency regions are presented. Each mode is presented in different intensity scale. First order modes $E_{2 \mathrm{~g}(1)}$ at $\approx 42 \mathrm{~cm}^{-1}$ and $E_{2 \mathrm{~g}(2)}$ at $\approx 1582 \mathrm{~cm}^{-1}$ are registered. To the best of our knowledge report of $E_{2 \mathrm{~g}(1)}$ mode at $\approx 42 \mathrm{~cm}^{-1}$ registered by the Raman spectroscopy is rareness. Second order $D^{*}$ and $G^{*}$ modes are also registered. $D^{*}$ band is strong, wide asymmetric towards lower frequencies. Weak spectral feature at $\approx 2450 \mathrm{~cm}^{-1}$ is registered, too.

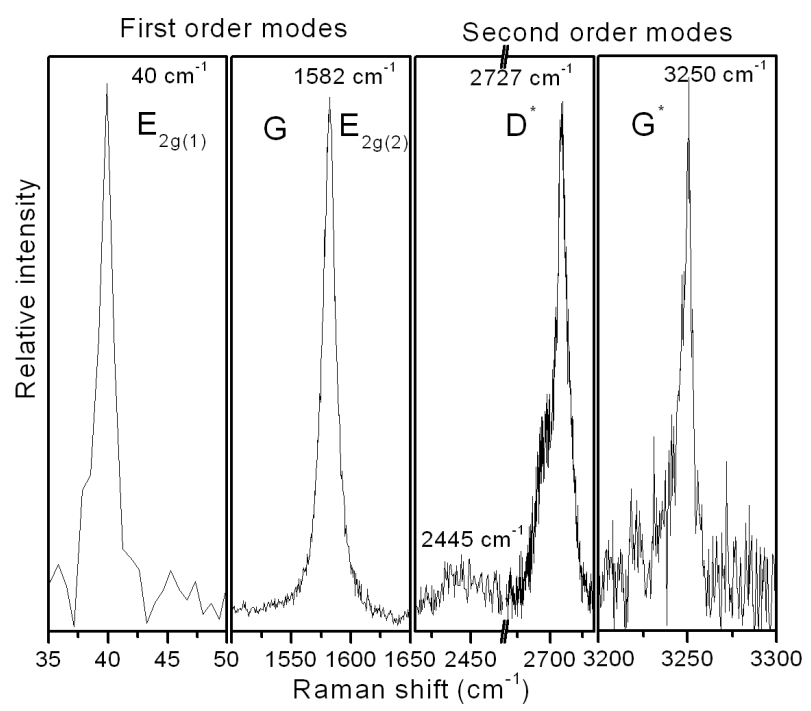

Fig. 3. First and second order Raman spectra of HOPG.

After optical characterization of HOPG we started with the mechanical exfoliation. We have measured the experimental Raman spectra of our samples after each step during the exfoliation. We followed change of $E_{2 \mathrm{~g}(2)}$ $(G), D^{*}$ and $G^{*}$ mode. The $G$ peak slightly up-shifts. Registration of low frequency $E_{2 g(1)}$ mode is connected to difficulties and spends a lot of time. In this spectral region we measured only HOPG Raman spectra. In this paper we present the Raman spectra in $D^{*}$ band spectral region i.e. $2600-3000 \mathrm{~cm}^{-1}$. Results for the $D^{*}$ band spectral region are presented in Fig. 4. For HOPG (top) we have band asymmetric towards lower frequencies with maximum at about $2727 \mathrm{~cm}^{-1}$. Arrow in Fig. 4 indicates lowering of layers number. Spare and position of this band change as number of layers decreases. Spectrum at the bottom of Fig. 4, with symmetric feature centered at $2700 \mathrm{~cm}^{-1}$, corresponds to graphene layer. 
It is obvious that graphene has a single $D^{*}$ peak. This band splits in multilayered case. Evolution of the electronic band with number of layers produces change in the Raman spectra. The $D^{*}$ peak in graphene is due to two phonons with opposite momentum in the highest optical branch near $K$ point of the Brillouin zone. Double resonance process links the phonon wave vectors to the electronic band structure [4]. In multilayer case the interaction of the graphene layers causes electronic band splitting. Phonons in the highest optical branch couple all electron bands. The resulting process involves phonons with different moment.

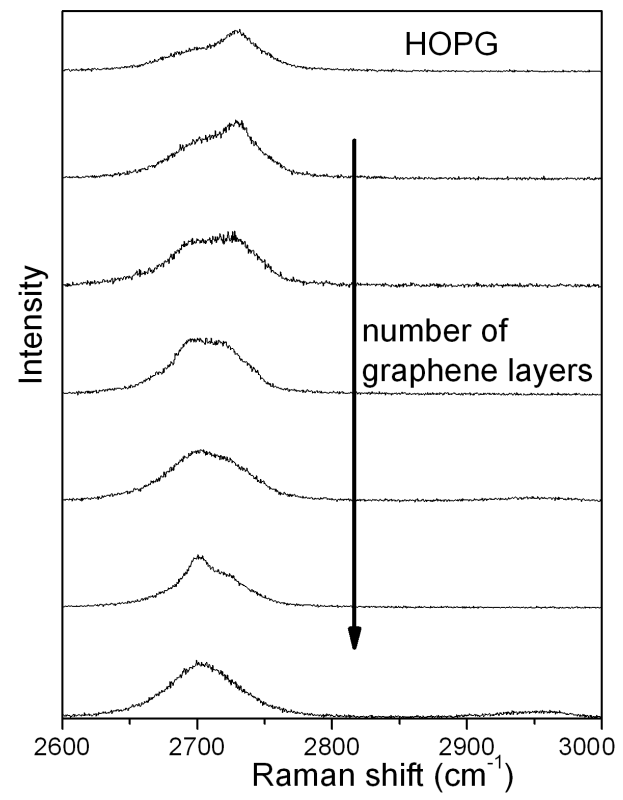

Fig. 4. Second order Raman spectra of $n$ GL films in $D^{*}$ band spectral region.

\section{Conclusion}

Results of room-temperature ellipsometric and Raman scattering studies of HOPG and multi-layer graphitic films are reported. All symmetry analysis predicted modes are registered.

IR active modes of HOPG are detected by ellipsometric measurements. In the first order Raman spectra we detected both Raman active modes of HOPG. Besides the most prominent $E_{2 g(2)}(G)$ mode of graphite, even a very low intensity $E_{2 \mathrm{~g}(1)}$ mode of $\mathrm{HOPG}$ is registered.

We studied $D^{*}$ peak spectral region with special attention. We confirm that the Raman spectroscopy clearly distinguishes a single layer from few layers case or HOPG. Graphene electronic structure and electron-phonon interaction that evolves with the number of layers can be viewed in their Raman spectra. This established the Raman spectroscopy as a very helpful nondestructive tool in identification of graphene layers and studies of their properties.

\section{Acknowledgments}

This work is supported by the Serbian Ministry of Science under project No. 141047. We acknowledge support from EU FP7 project Nanocharm.

\section{References}

[1] A. Gupta, G. Chen, P. Joshi, S. Tadigadapa, P.C. Eklud, Nano Lett. 6, 2267 (2006).

[2] J.S. Park, A. Reina, R. Saito, J. Kong, G. Dresselhaus, M.S. Dresselhaus, Carbon 47, 1303 (2009).

[3] Z.K. Wang, H.S. Lim, S.C. Ng, B. Ozyilmaz, M.H. Kouk, Carbon 46, 2133 (2009).

[4] A.C. Ferrari, J.C. Meyer, V. Scardaci, C. Casiraghi, M. Lazzeri, F. Mauri, S. Piscanec, D. Jiang, K.S. Novoselov, S. Roth, A.K. Geim, Phys. Rev. Lett. 97, 187401 (2006).

[5] C. Mapelli, C. Castiglioni, G. Zerbi, Phys. Rev. 60, 12710 (1999).

[6] J. Maultzsch, S. Reich, C. Thomsen, H. Requardt, P. Ordejon, Phys. Rev. Lett. 92, 075501 (2004).

[7] S. Reich, C. Thomsen, Philos. Trans. R. Soc. Lond. A 363, 2271 (2004).

[8] M. Mohr, J. Maultzsch, E. Dobardzic, S. Reich, I. Milosevic, M. Damjanovic, A. Bosak, M. Krisch, C. Thomsen, Phys. Rev. B 76, 035439 (2007).

[9] F. Tunistra, J.L. Koenig, J. Chem. Phys. 53, 1126 (1970).

[10] R.J. Nemanich, G. Lucovsky, Solid State Commun. 23, 117 (1977). 